\title{
Unilateral axilla-bilateral areola approach for thyroidectomy by da Vinci robot vs. open surgery in thyroid cancer: a retrospective observational study
}

\author{
Ye Zhang, Junze Du, Jing Ma, Jing Liu, Xiang Cui, Juan Yuan, Yi Zhang, Xiaowei Qi, Linjun Fan \\ Department of Breast and Thyroid Surgery, Southwest Hospital, Army Medical University, Chongqing, China \\ Contributions: (I) Conception and design: L Fan; (II) Administrative support: Y Zhang; (III) Provision of study materials or patients: Y Zhang, J Du, \\ J Ma, J Liu, X Cui, J Yuan; (IV) Collection and assembly of data: Y Zhang, X Qi; (V) Data analysis and interpretation: Y Zhang, X Qi, L Fan; (VI) \\ Manuscript writing: All authors; (VII) Final approval of manuscript: All authors. \\ Correspondence to: Linjun Fan; Xiaowei Qi. 29 Gaotanyan St, Shapingba District, Chongqing 400038, China. \\ Email: flj212@126.com; qxw9908@foxmail.com.
}

Background: To compare the efficacy and safety of unilateral axilla-bilateral areola (UABA) approach for
robot-assisted thyroidectomy with conventional open surgery in thyroid cancer patients.
Methods: The clinicopathological features and surgical outcomes of 194 thyroid cancer patients treated by
robotic surgery using the UABA approach and 217 patients treated by open surgery in our department from
January 2017 to August 2018 were analysed and compared.
Results: The operation time was longer in the robotic group than in the open surgery group. The patients'
satisfaction with neck appearance was higher in the robotic group than in the open surgery group $(91.2 \% v$ s.
$21.6 \%$, P $<0.01)$. After total thyroidectomy and central lymph node dissection, the incidence of postoperative
transient hypoparathyroidism and postoperative permanent hypoparathyroidism in the open surgery group
was $29.7 \%(44 / 148)$ and $6.8 \%(10 / 148)$, higher than that $[17.9 \%(27 / 151)$ and $2.0 \%(3 / 151)]$ in the robotic
group $(\mathrm{P}<0.05$ respectively). No significant difference was observed in the number of dissected lymph nodes
or postoperative serum thyroglobulin (TG) levels or incidence of transient or permanent hoarseness of voice
between the two groups. No recurrence or metastasis was found.

Conclusions: Compared with open surgery, UABA robotic surgery preserved the neck appearance and effectively reduced hypoparathyroidism by super-meticulous capsular dissection (SMCD).

\begin{abstract}
Keywords: Thyroid cancer surgery; open surgery; da Vinci robot; unilateral axilla-bilateral areola approach (UABA approach); super-meticulous capsular dissection (SMCD)
\end{abstract}

Submitted Nov 15, 2020. Accepted for publication Mar 03, 2021.

doi: 10.21037 /gs-20-831

View this article at: http://dx.doi.org/10.21037/gs-20-831

\section{Introduction}

Thyroid cancer is one of the most common malignancies in young women, with the increasing trend of annual incidence $(1,2)$. Compared with other malignancies, most thyroid cancer patients have a good prognosis, so they demand not only the radical removal of lesions but also better quality of life after surgery $(3,4)$. Endoscopic surgery is a good option for the treatment of thyroid diseases because it can well preserve the neck appearance. As one of the advanced endoscopic systems, the da Vinci robot has the advantages of $3 \mathrm{D}$ high-definition vision and a remote control that filters operator shaking; it also has flexible internal joints with 7 degrees of freedom, which is more conducive to meticulous operation compared with conventional open surgery $(5,6)$. Therefore, it has been widely used for thyroid surgery (7-10). However, it is unclear whether robotic thyroid surgery is superior to open surgery in terms of efficacy and complications (10-13). 
From January 2017 to August 2018, we treated 217 thyroid cancer patients by robotic surgery via a unilateral axilla-bilateral areola (UABA) approach (robotic group). Another 194 thyroid cancer patients who underwent open surgery in the same period served as the control group (open surgery group). The data pertaining to the efficacy and safety of the two groups were analyzed.

We present the following article in accordance with the STROBE reporting checklist (available at http://dx.doi. org/10.21037/gs-20-831).

\section{Methods}

\section{Case selection}

The patients who met the following criteria were involved: (I) at the age of 18 to 70 years; (II) diagnosed with thyroid cancer by preoperative fine-needle aspiration (FNA) or suspected malignancy by preoperative FNA confirmed by postoperative pathology; and (III) maximum tumor diameter $\leq 2 \mathrm{~cm}$ by ultrasound.

The exclusion criteria were as follows: (I) history of thyroid surgery or radiotherapy on the neck; (II) preoperative examination or intraoperative findings revealed the tumor invading esophagus, trachea, the recurrent laryngeal nerve, or large blood vessels on the neck; (III) preoperative examination showed suspected or definite metastasis of lateral lymph nodes; and (IV) preoperative examination indicated distant metastasis. The sample size of the study was estimated based on the data of pilot study prior to it.

The choice of operative methods: all patients were informed of the advantages and disadvantages of robotic surgery and open surgery, and made their own decisions before operation.

Quality control: according to the inclusion and exclusion criteria, we consecutively included 217 cases in robotic group and 194 cases in open surgery from 2017 to 2018. To avoid subjective bias, all robotic surgeries were performed by one surgeon, and all open surgeries were performed by another surgeon. Both surgeons had previously completed more than 1,000 thyroid cancer surgeries, and the surgeon in the robotic group had accomplished more than 500 robotic thyroid surgeries.

\section{Surgical methods}

\section{Open surgery}

The patient was in a supine and neck-hyperextended position. A 5-8 $\mathrm{cm}$ long arc incision was made in the front of the neck. The skin, subcutaneous tissue and the platysma were dissected in turn to free the flap along the deep surface of the platysma, upper to the upper edge of the thyroid cartilage, down to the sternal fossa, bilaterally towards the midline of the sternocleidomastoid muscle. The linea alba cervicalis and the strap muscle were dissected bilaterally to expose the thyroid gland. The thyroid isthmus, the inferior and superior thyroid vessels and the middle thyroid veins were removed using an ultrasound knife. The surgeon should try to preserve the parathyroid glands and their blood supply as much as possible. The recurrent laryngeal nerve was exposed and carefully preserved, and then Berry's ligament was dissected at the entry point of the laryngeal nerve to the larynx. The thyroid glands on one or both sides were removed; if necessary, a few glandular tissues at the entry point were reserved to protect the recurrent laryngeal nerve and superior parathyroid glands $(7,9,14)$. During the dissection of the central lymph nodes, the recurrent laryngeal nerve and the inferior parathyroid glands and were protected, and lymph nodes behind the nerve were removed, especially in central node dissection on the right.

\section{Robotic surgery}

The procedure for the robotic surgery with da Vinci $\mathrm{S}$ or $\mathrm{Si}$ robot (Silicon Valley, CA, USA) was previously described in the literature (15). A brief summary: the patient was placed in the supine position, and the shoulders were elevated with pads. The head was backwards hyperextended and the right upper limb was abducted by $90^{\circ}$ in order to expose the axilla, neck, and bilateral areolae. Mark the incision sites with ink on the right axilla and in the medial margin of bilateral areolae (1-1.5 cm incision), and draw the lines from each incision site to the superior border of the inner clavicle and right and left sternoclavicular joints to guide the trajectory of the trocars (Figure 1). The trocar was inserted and connected to the robotic arm, and a pressure of $6 \mathrm{mmHg}$ was maintained by insufflating $\mathrm{CO}_{2}$. The flaps were freed with the same operation as in open surgery. A special thyroid retractor was inserted through the neck skin to draw the strap muscle bilaterally to expose the surgical field. A cautery was used to dissect the linea alba cervicalis, remove the thyroid isthmus along the midline of the trachea and sever inferior thyroid vessels and middle thyroid vein. The posterior thyroid capsule was dissected super-meticulously using an ultrafine-tipped cautery [supermeticulous capsular dissection (SMCD)]. The thyroid gland was freed from posterior true thyroid capsule and the 


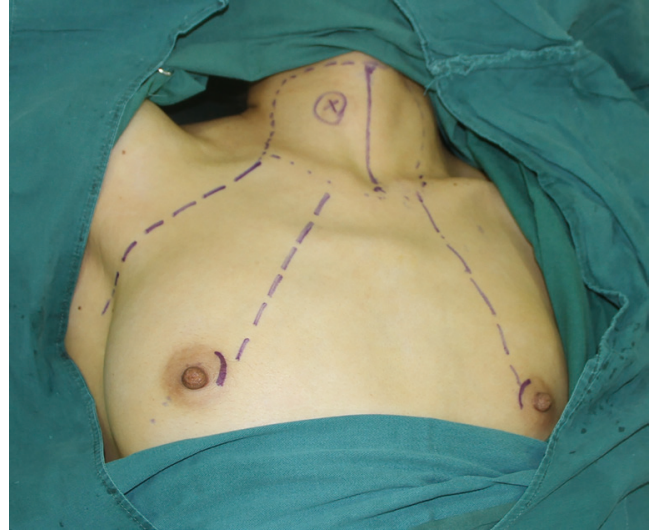

Figure 1 The incision sites were marked on the right axilla and in the medial margin of the bilateral areolae, and the line from each incision site to the superior border of the inner clavicle and right and left sternoclavicular joints, respectively, were used to guide the trajectory of the trocars.

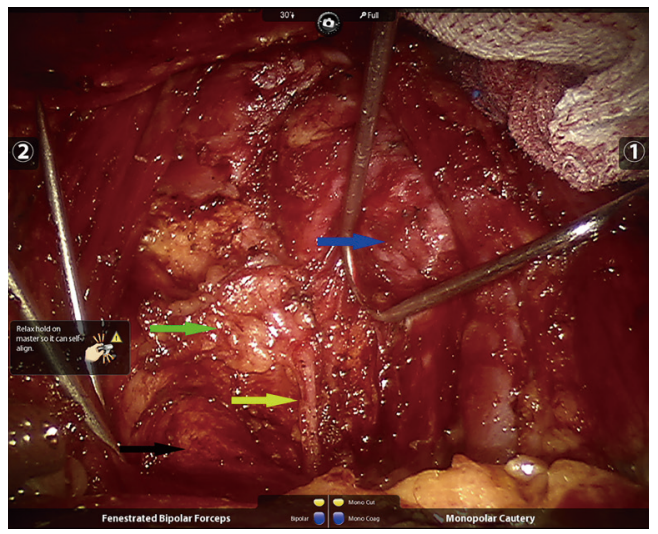

Figure 2 The strap muscle was drawn to the right and the trachea was drawn to the left using thyroid retractors to expose the right median area for central lymph node dissection. After dissection, the periphery structure was clear: the trachea (blue arrow), the right superior parathyroid gland (green arrow), the recurrent laryngeal nerve (yellow arrow), and the cervical artery (black arrow).

trachea to the entry point of the recurrent laryngeal nerve to the larynx. The recurrent laryngeal nerve was exposed, and attention was paid to preserving the nerve, as well as the superior parathyroid gland and its blood supply. Then, a total or near-total thyroidectomy was performed. For the central lymph node dissection on the right, the strap muscle was drawn to the right and the trachea was drawn to the left using thyroid retractors (Figure 2). For the central lymph

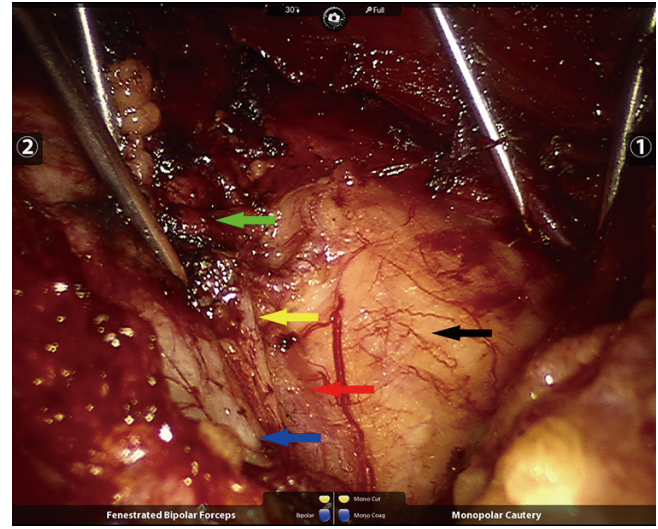

Figure 3 The strap muscle was drawn to the left and the trachea was drawn to the right using thyroid retractors to expose the left median area for central lymph node dissection. After dissection, the periphery structure was clear: the right superior parathyroid gland (green arrow), the recurrent laryngeal nerve (yellow arrow), the esophagus (red arrow), the trachea (blue arrow), and the left common carotid artery (black arrow).

node dissection on the left, the strap muscle was drawn to the left and the trachea was drawn to the right using thyroid retractors; thereafter, the central lymph nodes were removed by a cautery (Figure 3).

\section{Postoperative treatment and follow-up}

The patient's voice was observed postoperatively. The color and volume of the drainage fluid were recorded daily, and when the drainage fluid turned pale red and the daily volume was less than $15 \mathrm{~mL}$, the drainage tube was removed. The patients were followed up in the clinic at postoperative months 1,3 and 6 and then rechecked every six months thereafter. The follow-up evaluations included a thyroid function test, determination of thyroglobulin (TG), serum calcium and parathyroid hormone (PTH) levels, and cervical ultrasound. According to laboratory testing results, serum calcium level $<2.2 \mathrm{mmol} / \mathrm{mL}$ is defined as hypocalcemia. PTH $<15 \mathrm{pg} / \mathrm{mL}$ is defined as hypoparathyroidism. If PTH returns to normal within 6 months, the patient was diagnosed with transient hypoparathyroidism, otherwise, permanent hypoparathyroidism. Any bleeding that requires surgical intervention is considered as postoperative haemorrhage.

The study was conducted in accordance with the Declaration of Helsinki (as revised in 2013). The study 
was approved by the Ethics Committee of the Southwest Hospital (IRB No. ChiCTR2000033674), which waived the requirement for informed consent of clinicopathologic data due to the retrospective nature of this study.

\section{Statistical analysis}

All data were analyzed using SPSS 25.0 software. Continuous variables were expressed as mean \pm SD . The count data were expressed as absolute values and percentages. The measurement data were analyzed by $t$-test or the Mann-Whitney $\mathrm{U}$ test, and the count data were analyzed by $\chi^{2}$ test or the Jonckheere-Terpstra test. A value of $\mathrm{P}<0.05$ was considered significantly different.

\section{Results}

\section{Comparative analysis of clinicopathological features}

No significant differences were observed in patients' age, gender, BMI, location and size of tumor, number of lesions, pathological type or scope of resection between the open surgery group and the robotic group (Table 1).

\section{Number of dissected lymph nodes and operation time}

Postoperative pathology demonstrated no significant difference in the number of dissected lymph nodes or metastatic lymph nodes between the two groups, regardless of whether the central node dissection was unilateral or bilateral. The robotic group showed significantly longer operation time compared with open surgery group (Table 2).

\section{Satisfaction with the appearance}

The survey on patient satisfaction with neck appearance was conducted 6 months after surgery based on the method described by Ji et al. (16). The patients' satisfaction with their neck appearance was $91.2 \%$ in the robotic group, which was significantly higher than $21.6 \%$ in the open surgery group $(\mathrm{P}<0.01$, Table 3$)$.

\section{Efficacy and complications}

For the patients who underwent total thyroidectomy and unilateral central node dissection, the concentration of TG without interference of thyroid stimulating hormones was $0.502 \pm 1.43 \mathrm{ng} / \mathrm{mL}$ in the open surgery group and
$0.700 \pm 1.73 \mathrm{ng} / \mathrm{mL}$ in the robotic group at one month after surgery, suggesting no significant difference between the two groups $(\mathrm{P}=0.073)$. For the patients who underwent total thyroidectomy and bilateral central node dissection, the concentration of TG was $0.545 \pm 1.45 \mathrm{ng} / \mathrm{mL}$ in the open surgery group and $0.603 \pm 1.11 \mathrm{ng} / \mathrm{mL}$ in the robotic group at 1 month after surgery, suggesting no significant difference between the two groups $(\mathrm{P}=0.090)$.

The incidence of postoperative hypocalcemia in the open surgery group was $27.3 \%(53 / 194)$, which was significantly higher than the incidence of $14.3 \%(31 / 217)$ in the robotic group $(\mathrm{P}<0.01)$. In the open surgery group, the incidence of transient hypoparathyroidism was $29.7 \%$ (44/148) in patients who underwent total thyroidectomy and central lymph node dissection, which was significantly higher than the incidence of $17.9 \%(27 / 151)$ in the robotic group $(\mathrm{P}=0.016)$. In the open surgery group, the incidence of permanent hypoparathyroidism was $6.8 \%(10 / 148)$ in patients who underwent total thyroidectomy and central lymph node dissection, significantly higher than $2.0 \%$ (3/151) in the robotic group $(\mathrm{P}=0.043)$. Transient hoarseness of voice occurred in 2 cases of open surgery group (1.0\%) and 1 case of robotic group $(0.5 \%)$, indicating no significant difference between the two groups $(\mathrm{P}=0.604)$. One patient in the open surgery group had permanent hoarseness of voice, while none of the patients in the robotic group had this change, indicating no significant difference between the two groups $(\mathrm{P}=0.472)$. Postoperative hemorrhage occurred in four cases of open surgery group and in one case of robotic group, indicating no significant difference between the two groups $(\mathrm{P}=0.193)$. Postoperative wound infection occurred in five patients $(2.6 \%)$ in the open surgery group and in four patients $(1.8 \%)$ in the robotic group, indicating no significant difference between the two groups $(\mathrm{P}=0.741)$. One patient in the open surgery group had a chyle fistula $(0.5 \%)$, and none was observed in the robotic group, indicating no significant difference between the two groups $(\mathrm{P}=0.472)$. All complications are detailed in Table 4. The patients in the open surgery group were followed up for median 23 months and the patients in the robotic group were followed up for median 22 months. No recurrence or metastasis was observed in the two groups during the follow-up.

\section{Discussion}

The da Vinci robot system has been used for surgical treatment of thyroid cancer $(7,14)$ due to its high-definition 
Table 1 Clinicopathological features of the two groups

\begin{tabular}{|c|c|c|c|}
\hline Clinicopathological features & Open surgery group $(n=194), n(\%)$ & Robotic group (n=217), n (\%) & $P$ value \\
\hline Gender & & & 0.528 \\
\hline Female & $150(77.3)$ & $162(74.7)$ & \\
\hline Male & $44(22.7)$ & $55(25.3)$ & \\
\hline Tumor location & & & 0.362 \\
\hline Left & $68(35.0)$ & $83(38.2)$ & \\
\hline Right & $95(49.0)$ & $112(51.6)$ & \\
\hline Isthmus & $5(2.6)$ & $3(1.4)$ & \\
\hline Single & $148(76.3)$ & $156(71.9)$ & \\
\hline Multiple & $46(23.7)$ & $61(28.1)$ & \\
\hline Tumor size & & & 0.921 \\
\hline$<10 \mathrm{~mm}$ & $93(47.9)$ & $106(52.1)$ & \\
\hline$\geq 10 \mathrm{~mm}$ & $101(52.1)$ & $111(47.9)$ & \\
\hline Pathological type & & & 0.775 \\
\hline Papillary carcinoma & $189(97.4)$ & $212(97.7)$ & \\
\hline Follicular carcinoma & $3(1.6)$ & $4(1.8)$ & \\
\hline $\begin{array}{l}\text { Total thyroidectomy + bilateral central node } \\
\text { dissection }\end{array}$ & $47(24.2)$ & $35(16.1)$ & \\
\hline RAl ablation & 41 & 44 & 0.830 \\
\hline
\end{tabular}

BMI, body mass index; RAI, radioiodine.

3D field of view, remote control operation that filters hand shaking, and internal joint instruments $(11,12)$. However, whether robotic surgery is superior to conventional open surgery is still controversial because of its high cost and clinical outcomes $(8,12,13,17,18)$. To our acknowledgement, this is the first study to compare UABA robotic surgery with open surgery, and our results indicate that UABA robotic thyroidectomy can preserve the neck appearance and effectively reduce hypoparathyroidism by SMCD.
Most robotic thyroid surgeries adopt the transaxillary approach (TAA) or the bilateral axilla-bilateral areola approach (BABA) (19-22). When using the UABA approach, we reduce one axillary incision and the corresponding subcutaneous channel and move the only axillary incision from the anterior axillary line to the midaxillary line, which is more occult. This approach not only reduces the trauma but also improves the cosmetic effect. The external retractor was used for a better exposure of surgical field, and only 
Table 2 Comparison of dissected lymph node number and operation time between the two groups

\begin{tabular}{lcc}
\hline Variables & Open surgery group $(\mathrm{n}=194)$ & Robotic group $(\mathrm{n}=217)$ \\
\hline Dissected lymph nodes & & $\mathrm{P}$ value \\
Unilateral lobectomy + unilateral central node dissection & $5.93 \pm 3.85$ & $5.73 \pm 3.24$ \\
Total thyroidectomy + unilateral central node dissection & $7.22 \pm 9.36$ & $6.47 \pm 4.15$ \\
Total thyroidectomy + bilateral central node dissection & $9.30 \pm 4.80$ & $9.49 \pm 4.25$ \\
Metastatic lymph nodes & & 0.114 \\
Unilateral lobectomy + unilateral central node dissection & $0.76 \pm 1.69$ & $1.10 \pm 1.60$ \\
Total thyroidectomy + unilateral central node dissection & $1.82 \pm 3.54$ & $1.32 \pm 2.02$ \\
Total thyroidectomy + bilateral central node dissection & $1.68 \pm 2.33$ & $2.54 \pm 3.66$ \\
Operation time (min) & & 0.092 \\
Unilateral lobectomy + unilateral central node dissection & $96.48 \pm 52.10$ & 0.224 \\
Total thyroidectomy + unilateral central node dissection & $99.27 \pm 57.88$ & $130.73 \pm 38.18$ \\
Total thyroidectomy + bilateral central node dissection & $93.51 \pm 36.23$ & $152.52 \pm 37.79$ \\
\hline
\end{tabular}

Table 3 Patient satisfaction with neck appearance in the two groups

\begin{tabular}{lcc}
\hline Patient satisfaction & Open surgery group $(\mathrm{n}=194), \mathrm{n}(\%)$ & Robotic group $(\mathrm{n}=217), \mathrm{n}(\%)$ \\
\hline High & $42(21.6)$ & $198(91.2)$ \\
Moderate & $145(74.7)$ & $19(8.8)$ \\
Unsatisfied & $7(3.6)$ & 0 \\
\hline
\end{tabular}

Table 4 Comparison of postoperative thyroglobulin levels and complications in the two groups

\begin{tabular}{|c|c|c|c|}
\hline Parameters & $\begin{array}{l}\text { Open surgery group }(n=194) \\
n(\%)\end{array}$ & $\begin{array}{l}\text { Robotic group ( } \mathrm{n}=217) \text {, } \\
\mathrm{n}(\%)\end{array}$ & $P$ value \\
\hline \multicolumn{4}{|l|}{ Thyroglobulin } \\
\hline Total thyroidectomy + bilateral central node dissection & $0.545 \pm 1.45$ & $0.603 \pm 1.11$ & 0.090 \\
\hline Transient & $44 / 148(29.7)$ & $27 / 151(17.9)$ & 0.016 \\
\hline Permanent & $10 / 148(6.8)$ & $3 / 151(2.0)$ & 0.043 \\
\hline \multicolumn{4}{|l|}{ Hoarseness of voice } \\
\hline Postoperative hemorrhage & $4(2.1)$ & $1(0.5)$ & 0.193 \\
\hline Postoperative infection & $5(2.6)$ & $4(1.8)$ & 0.741 \\
\hline Chyle fistula & $1(0.5)$ & 0 & 0.472 \\
\hline
\end{tabular}


three robotic arms are used to avoid interaction between the arms and the instruments. Our previous research has confirmed that the UABA approach is safe, effective and minimally invasive for robotic thyroid surgery (15).

We analyzed the clinical data of 217 thyroid cancer patients who underwent robotic surgery using the UABA approach and 194 thyroid cancer patients who underwent conventional open surgery. The results showed that there was no significant difference in postoperative TG level and the number of dissected lymph nodes between the two groups, indicating that robotic surgery can achieve the same efficacy as open surgery in the treatment of thyroid cancer if the surgeon carefully chooses the indications. However, via the UABA approach, the incisions are occult, with little effect on neck appearance after surgery, so patient satisfaction is significantly higher in the robotic group than in the open surgery group after surgery. In the open surgery approach for thyroid cancer, incisions are located on the front of the neck, leaving significant scars after surgery in most cases. Compared with Caucasians, incisions on the neck are more likely to leave scars in Asian patients postoperatively. Robotic thyroid surgery using the non-transcervical approach can avoid scars on the neck and preserve neck appearance after thyroid surgery, so it is widely used in Asian countries such as South Korea and China (23,24).

Anatomically, the parathyroid glands have a small volume, great variation in position and extremely small blood vessels, so hypoparathyroidism frequently occurs after thyroid cancer surgery and is regarded as one of the biggest challenges in the clinic (25). The 3D high-definition magnified field of view and internal joint instruments in robotic surgery help surgeons operate more precisely; theoretically, robotic surgery can protect the parathyroid glands better and reduce postoperative complications. However, in the literature, disparity exists in the incidences of complications after robotic surgery and open surgery; e.g., in some studies, the robotic thyroid surgery group showed a similar or higher incidence of hypoparathyroidism (10-12). In our study, the incidences of hypocalcemia and hypoparathyroidism in the robotic group were significantly lower than those in the open surgery group. This may be because the surgeon used the technical advantages of da Vinci robot to create SMCD in the robotic surgeries $(15,26)$. By means of SMCD, if compact, embedded or subcapsular parathyroid glands were observed, the surgeon could dissect the true thyroid capsule and preserve the parathyroid glands together with the surrounding true capsule to preserve the blood supply to the parathyroid glands. During conventional meticulous capsule dissection of the open surgery, because of the vision limits of human eyes and the range of motion for common surgical devices, the thyroid gland is removed closely adhered to the true thyroid capsule, and the parathyroid glands are preserved as much as possible, causing the difference in the incidence of postoperative hypoparathyroidism between the two groups. It is believed that there is no significant difference in efficacy between robotic thyroid surgery and open surgery $(11,27)$. The robotic thyroid surgery can better preserve neck appearance, so young patients and female patients tend to choose robotic surgery (27). In our study, we performed SMCD to protect parathyroid function in robotic thyroid surgery, superior to open surgery in reducing the incidence of complications. Considering this, in addition to aesthetic factors, most of the patients choose robotic surgery because robotic surgery can better protect the parathyroid glands and reduce the incidence of hypoparathyroidism. The patients' age and gender showed no significant difference between robotic and open surgery group in our study.

Studies have shown that the learning curve for robotic thyroid surgery requires more than 50 cases $(7,28-31)$. However, the surgeon should be more experienced and proficient in addressing complicated situations in thyroid surgery. Our previous study of 500 cases treated by the same surgeon showed that the latter 250 cases had significantly fewer complications than the former 250 cases (15). To avoid the influence of the surgeon's proficiency on surgical outcomes, the surgeon in the robotic group and the surgeon in the open surgery group had both completed more than 1,000 thyroid cancer surgeries, and the surgeon in the robotic group had accomplished more than 500 robotic thyroid cancer surgeries in particular. Nevertheless, the operation time in the robotic group was significantly longer than that in the open surgery group, which may be because robotic surgery requires more preparation procedures, such as placing sterile plastic sleeves and connecting the robotic arms. Additionally, it takes more time to establish the operation space, place the gauze, remove the specimens, and rinse and suction the surgical field in robotic surgery than in open surgery.

The limitations of our study were as follow: (I) to avoid the influence of the surgeon's proficiency on surgical outcomes, we enrolled the patients treated by the most experienced surgeon in the robotic group and in the open surgery group, respectively. Even so, the selection bias is inevitable. (II) We evaluated the vocal cord activity by 
voice changes (hoarseness of voice), which is less reliable than the laryngoscopy. (III) Our study was a single-center clinical trial with a small sample size, so more multicenter randomized controlled studies are needed for further verification.

In conclusion, robotic surgery using the UABA approach can achieve the same efficacy as open surgery in the treatment of thyroid cancer, with lower incidence rates of transient and permanent hypoparathyroidism and a better cosmetic effect of the neck; therefore, the UABA approach is a safe, reliable and feasible approach with good cosmetic effects.

\section{Acknowledgments}

We thank Ms Junlan Liu from the Southwest Hospital of Army Medical University for her help in language editing and proofreading.

Funding: This work was supported by the Science and Technology Innovation Project for Social Livelihood of Chongqing (grant number cstc2019jscx-msxmX0284, cstc2019jscx-msxmX0140) and Clinical Research Funding of Southwest Hospital (SWH2016JSTSYB-41).

\section{Footnote}

Reporting Checklist: The authors have completed the STROBE reporting checklist. Available at http://dx.doi. org/10.21037/gs-20-831

Data Sharing Statement: Available at http://dx.doi. org/10.21037/gs-20-831

Peer Review File: Available at http://dx.doi.org/10.21037/gs20-831

Conflicts of Interest: All authors have completed the ICMJE uniform disclosure form (available at http://dx.doi. org/10.21037/gs-20-831). The authors have no conflicts of interest to declare.

Ethical Statement: The authors are accountable for all aspects of the work in ensuring that questions related to the accuracy or integrity of any part of the work are appropriately investigated and resolved. The study was conducted in accordance with the Declaration of Helsinki (as revised in 2013). The study was approved by the Ethics Committee of the Southwest Hospital (IRB No.:
ChiCTR2000033674), which waived the requirement for informed consent of clinicopathologic data due to the retrospective nature of this study.

Open Access Statement: This is an Open Access article distributed in accordance with the Creative Commons Attribution-NonCommercial-NoDerivs 4.0 International License (CC BY-NC-ND 4.0), which permits the noncommercial replication and distribution of the article with the strict proviso that no changes or edits are made and the original work is properly cited (including links to both the formal publication through the relevant DOI and the license). See: https://creativecommons.org/licenses/by-nc-nd/4.0/.

\section{References}

1. Siegel RL, Miller KD, Jemal A. Cancer statistics, 2018. CA Cancer J Clin 2018;68:7-30.

2. Chen $W$, Zheng R, Baade PD, et al. Cancer statistics in China, 2015. CA Cancer J Clin 2016;66:115-32.

3. Roman BR, Morris LG, Davies L. The thyroid cancer epidemic, 2017 perspective. Curr Opin Endocrinol Diabetes Obes 2017;24:332-6.

4. Carling T, Udelsman R. Thyroid cancer. Annu Rev Med 2014;65:125-37.

5. Fan LJ, Jiang J. Present and future of robot-assisted endoscopic thyroid surgery. Chin Med J (Engl) 2012;125:926-31.

6. Rabinovics N, Aidan P. Robotic transaxillary thyroid surgery. Gland Surg 2015;4:397-402.

7. Kim EB, Cho JW, Lee YM, et al. Postsurgical Outcomes and Surgical Completeness of Robotic Thyroid Surgery: A Single Surgeon's Experience on 700 Cases. J Laparoendosc Adv Surg Tech A 2018;28:540-5.

8. Bae DS, Koo DH. A Propensity Score-matched Comparison Study of Surgical Outcomes in Patients with Differentiated Thyroid Cancer After Robotic Versus Open Total Thyroidectomy. World J Surg 2019;43:540-51.

9. Chai YJ, Suh H, Woo JW, et al. Surgical safety and oncological completeness of robotic thyroidectomy for thyroid carcinoma larger than $2 \mathrm{~cm}$. Surg Endosc 2017;31:1235-40.

10. Pan JH, Zhou H, Zhao XX, et al. Robotic thyroidectomy versus conventional open thyroidectomy for thyroid cancer: a systematic review and meta-analysis. Surg Endosc 2017;31:3985-4001.

11. Shan L, Liu J. Meta-analysis Comparison of Bilateral AxilloBreast Approach Robotic Thyroidectomy and Conventional 
Thyroidectomy. Surg Innov 2019;26:112-23.

12. Wang YC, Liu K, Xiong JJ, et al. Robotic thyroidectomy versus conventional open thyroidectomy for differentiated thyroid cancer: meta-analysis. J Laryngol Otol 2015;129:558-67.

13. Paek SH, Kang KH, Kang H, et al. Comparison of postoperative surgical stress following robotic thyroidectomy and open thyroidectomy: a prospective pilot study. Surg Endosc 2016;30:3861-6.

14. Kim WW, Jung JH, Park HY. A single surgeon's experience and surgical outcomes of 300 robotic thyroid surgeries using a bilateral axillo-breast approach. J Surg Oncol 2015;111:135-40.

15. Liu P, Zhang Y, Qi X, et al. Unilateral Axilla-Bilateral Areola Approach for Thyroidectomy by da Vinci Robot: 500 Cases Treated by the Same Surgeon. J Cancer 2019;10:3851-9.

16. Ji YB, Song CM, Bang HS, et al. Long-term cosmetic outcomes after robotic/endoscopic thyroidectomy by a gasless unilateral axillo-breast or axillary approach. J Laparoendosc Adv Surg Tech A 2014;24:248-53.

17. Berber E, Bernet V, Fahey TJ 3rd, et al. American Thyroid Association Statement on Remote-Access Thyroid Surgery. Thyroid 2016;26:331-7.

18. Aidan P, Arora A, Lorincz B, et al. Robotic Thyroid Surgery: Current Perspectives and Future Considerations. ORL J Otorhinolaryngol Relat Spec 2018;80:186-94.

19. Ryu HR, Lee J, Park JH, et al. A comparison of postoperative pain after conventional open thyroidectomy and transaxillary single-incision robotic thyroidectomy: a prospective study. Ann Surg Oncol 2013;20:2279-84.

20. Seup Kim B, Kang KH, Park SJ. Robotic modified radical neck dissection by bilateral axillary breast approach for papillary thyroid carcinoma with lateral neck metastasis. Head Neck 2015;37:37-45.

21. Kim WW, Kim JS, Hur SM, et al. Is robotic surgery superior to endoscopic and open surgeries in thyroid

Cite this article as: Zhang Y, Du J, Ma J, Liu J, Cui X, Yuan J, Zhang Y, Qi X, Fan L. Unilateral axilla-bilateral areola approach for thyroidectomy by da Vinci robot $v s$. open surgery in thyroid cancer: a retrospective observational study. Gland Surg 2021;10(4):1291-1299. doi: 10.21037/gs-20-831 cancer? World J Surg 2011;35:779-84.

22. Kim BS, Kang KH, Kang H, et al. Central neck dissection using a bilateral axillo-breast approach for robotic thyroidectomy: comparison with conventional open procedure after propensity score matching. Surg Laparosc Endosc Percutan Tech 2014;24:67-72.

23. Tae K, Ji YB, Song CM, et al. Robotic and Endoscopic Thyroid Surgery: Evolution and Advances. Clin Exp Otorhinolaryngol 2019;12:1-11.

24. Chang EHE, Kim HY, Koh YW, et al. Overview of robotic thyroidectomy. Gland Surg 2017;6:218-28.

25. Zhu J, Tian W, Xu Z, et al. Expert consensus statement on parathyroid protection in thyroidectomy. Ann Transl Med 2015;3:230.

26. Qi X, Du J, Liu H, et al. First report of in-situ preservation of a subcapsular parathyroid gland through super-meticulous capsular dissection during robotic radical thyroidectomy. Surg Oncol 2019;28:9-13.

27. Lang BH, Wong CK, Tsang JS, et al. A systematic review and meta-analysis evaluating completeness and outcomes of robotic thyroidectomy. Laryngoscope 2015;125:509-18.

28. Kim H, Kwon H, Lim W, et al. Quantitative Assessment of the Learning Curve for Robotic Thyroid Surgery. J Clin Med 2019;8:402.

29. Kim WW, Jung JH, Park HY. The Learning Curve for Robotic Thyroidectomy Using a Bilateral Axillo-Breast Approach From the 100 Cases. Surg Laparosc Endosc Percutan Tech 2015;25:412-6.

30. Park JH, Lee J, Hakim NA, et al. Robotic thyroidectomy learning curve for beginning surgeons with little or no experience of endoscopic surgery. Head Neck 2015;37:1705-11.

31. Lörincz BB, Busch CJ, Möckelmann N, et al. Initial learning curve of single-incision transaxillary robotic hemi- and total thyroidectomy--A single team experience from Europe. Int J Surg 2015;18:118-22. 